\title{
Hormone Therapy in Prostate Cancer
}

\author{
Geoffrey M. Currie ${ }^{1,2}$, Matthew Haase ${ }^{3}$, Rashid Hashmi ${ }^{3}$, and Hosen Kiat ${ }^{1,2}$ \\ ${ }^{I}$ Faculty of Science, Charles Sturt University, Wagga Wagga, New South Wales, Australia; ${ }^{2}$ Australian School of Advanced Medicine, \\ Macquarie University, Sydney, Australia; and ${ }^{3}$ RIL/Imed, Wagga Wagga, Australia
}

The role of nuclear medicine diagnostic bone scanning is well established in prostate cancer. This case provides an insight into the specific role that bone scanning plays in monitoring response to hormone therapy and an example of significant global skeletal response. The case highlights the remarkable efficacy of timely hormone therapy in high-grade prostate cancer with widespread bony metastasis. In addition, the range of hormone therapy currently available for clinical application in the management of metastatic prostate cancer is detailed. Finally, the case represents an incidental diagnosis of prostate cancer after evaluation of nonspecific symptoms.

Key Words: prostate carcinoma; hormone ablation therapy; $\mathrm{GnRH}$ agonist; ADT; bone scintigraphy

J Nucl Med Technol 2013; 41:49-51

DOI: 10.2967/jnmt.112.118034

W. e present a case study that provides an insight into the specific role that bone scanning plays in monitoring response to hormone therapy. The case highlights the remarkable efficacy of timely hormone therapy in high-grade prostate cancer with widespread bony metastasis.

\section{CASE REPORT}

An 83-y-old man presented for evaluation of reported weight loss and constipation. These symptoms were initially assessed by CT of the chest, abdomen, and pelvis. The findings were unremarkable, although a left renal staghorn calculus was noted. Despite no previous history of neoplasia, extensive sclerotic lesions were noted throughout the axial skeleton. This finding, combined with the observation of an irregular prostate contour (without enlargement), raised suspicion of previously undiagnosed widespread metastatic prostate carcinoma. Subsequent follow-up revealed a prostate-specific antigen (PSA) level of $823 \mu \mathrm{g} / \mathrm{L}$ (normal, $<4 \mu \mathrm{g} / \mathrm{L}$ ) and bone scan findings consistent with widespread skeletal metastases (Fig. 1). Subsequent prostate biopsy confirmed aggressive prostate carcinoma with a Gleason score of

\footnotetext{
Received Dec. 2, 2012; revision accepted Jan. 28, 2013.

For correspondence or reprints contact: Geoffrey M. Currie, P.O. Box U102, Charles Sturt University, Wagga Wagga 2650, New South Wales, Australia.

E-mail: gcurrie@csu.edu.au

Published online Feb. 12, 2013.

COPYRIGHT (C 2013 by the Society of Nuclear Medicine and Molecular Imaging, Inc.
}

9 (1). The patient commenced hormone therapy using bicalutamide (150 mg; Cosudex [AstraZeneca]) daily (oral tablet) and leuprorelin (30 mg; Lucrin [AbbVie]) depot (intramuscular injection) every 4 mo. The medications were well tolerated. At week 4, goserelin (Zoladex; AstraZeneca), a 10.8-mg subcutaneous implant (1 dose at 3 monthly intervals), was added to the patient's antitumor therapeutic armamentarium.

At 5 mo after the commencement of therapy, the PSA level had normalized to $0.39 \mathrm{ug} / \mathrm{L}$. It dropped further to $0.11 \mu \mathrm{g} / \mathrm{L}$ at $10 \mathrm{mo}$, corresponding to scintigraphic resolution of bony metastatic changes on the follow-up nuclear medicine bone scan (Fig. 2).

\section{DISCUSSION}

\section{Hormone Therapy}

The approach taken in the treatment of prostate cancer will depend on several factors, including the clinical presentation, Gleason score, and PSA (2). For patients with metastatic spread of prostate cancer, such as the patient in this case, the treatment of choice is androgen-deprivation therapy (ADT) $(2,3)$. ADT has been reported to lead to remission in as many as $80 \%$ of patients with advanced prostate carcinoma $(3,4)$. ADT is used in advanced prostate cancer to relieve pain, prevent pathologic fractures, and prevent neurologic complications (4). Typically, androgen deprivation is achieved through 3 mechanisms in prostate carcinoma patients, gonadotrophin-releasing hormone $(\mathrm{GnRH})$ agonists, antiandrogens, and $\mathrm{GnRH}$ antagonists.

$\mathrm{GnRH}$ agonists (goserelin, leuprorelin, and triptorelin) are considered the leading approach to ADT in prostate carcinoma (5). GnRH is released in pulses from the hypothalamus to stimulate pituitary release of luteinizing hormone, which, in turn, stimulates the secretion of testosterone in the testes $(5,6)$. GnRH agonists decrease testosterone levels by overstimulation, downregulation, and eventually receptor desensitization (5-7). There is, however, an initial flare or surge in testosterone production before suppression is effected (5). Unfortunately, testosterone suppression also increases the risk of osteoporosis and fracture (loss of bone mineral density), diabetes (altered glucose tolerance), and cardiovascular disease (7). Some of the more common adverse effects of ADT include weight gain, erectile dysfunction, decreased libido, gynecomastia, insomnia, sweats or chills, and gastrointestinal disturbances (7).

Antiandrogens (bicalutamide, flutamide, and nilutamide) are nonsteroidal inhibitors of androgen receptors $(6,7)$. The 


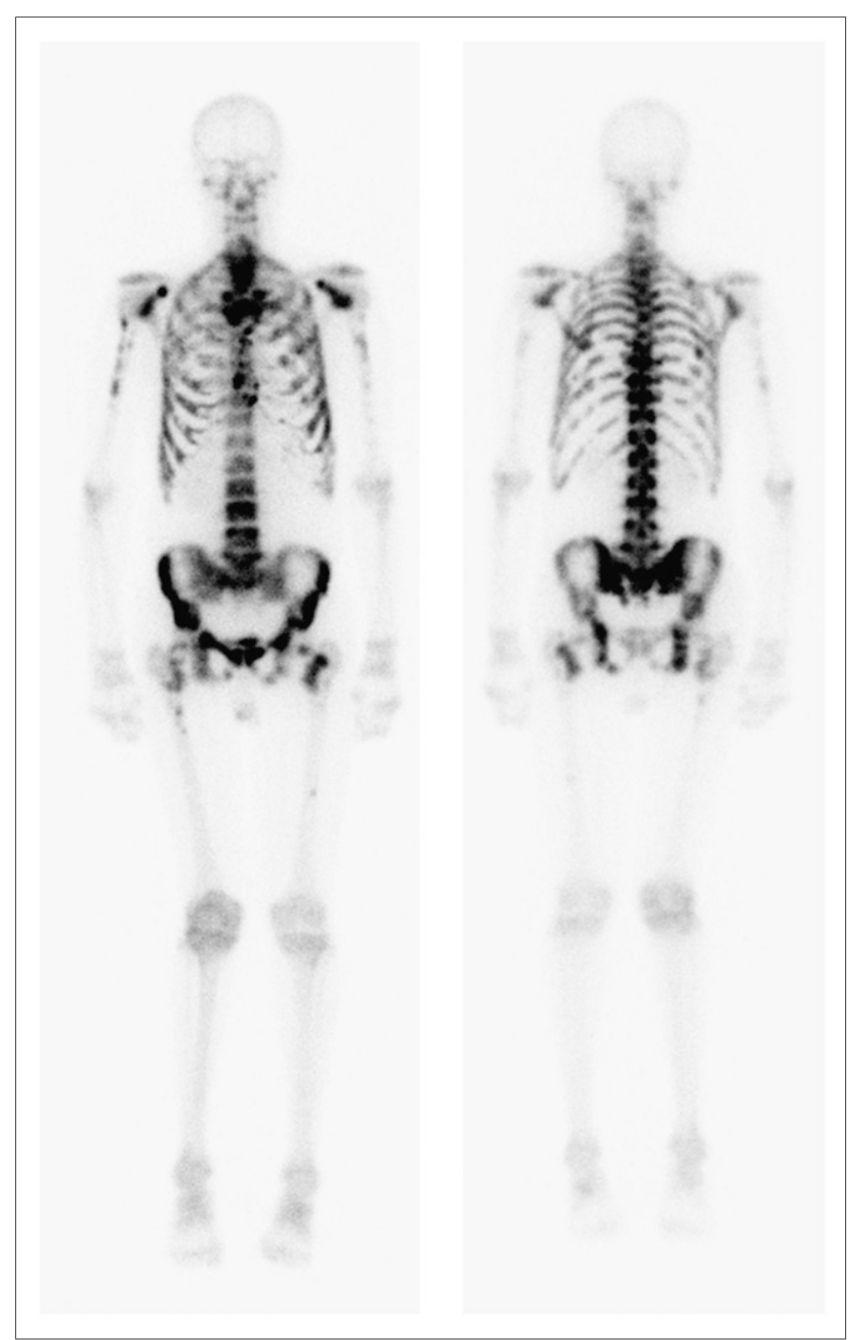

FIGURE 1. Baseline nuclear medicine bone scan demonstrating widespread metastatic disease associated with prostate carcinoma.

advantage of this class of ADT medications is that testosterone levels are not suppressed, and thus the associated risks discussed above are largely avoided $(6,7)$. That is, antiandrogens inhibit conversion of testosterone to dihydrotestosterine (metabolite) and prevent both from binding to the androgen receptor, without suppressing serum testosterone levels. The flare in serum testosterone associated with GnRH agonists means that they should be avoided as monotherapy in patients with severe pain or neurologic problems. Antiandrogens can be used as adjunctive therapy to block the flare (4).

GnRH antagonists (degarelix) have only recently received attention in the literature. GnRH agonists can cause an early increase in testosterone levels which, in turn, can promote tumor spread and worsen symptoms (2). GnRH antagonists can be used before the GnRH agonist therapy to block testosterone increases $(2,7)$. GnRH antagonists can also be used as an alternative approach to androgen deprivation by inhibiting gonadotrophin production, leading to a reduction in the synthesis of androgens in the testes (7).

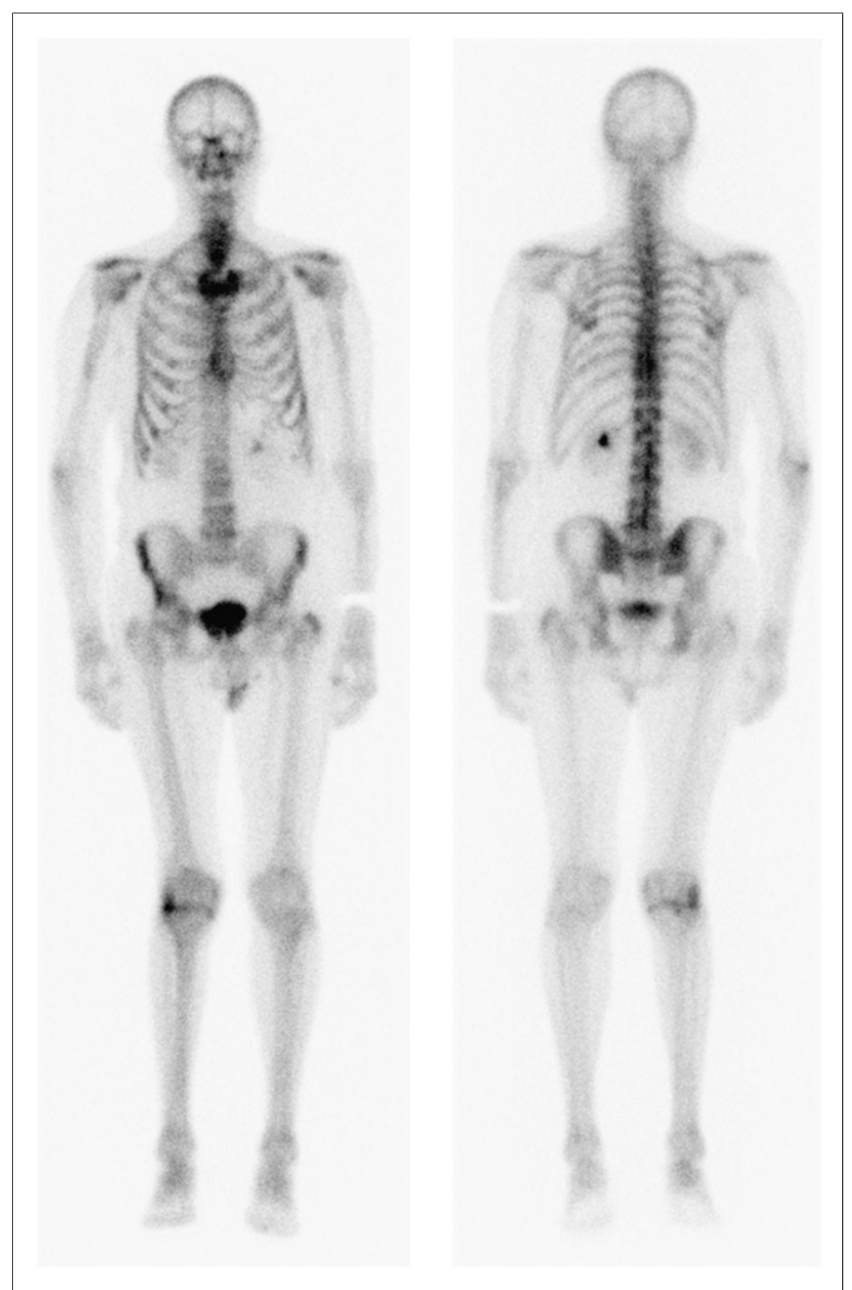

FIGURE 2. Follow-up nuclear medicine bone scan demonstrating remission of metastatic disease after hormone ablation therapy.

\section{Bone Scan}

ADT has been shown to result in remission in as many as $80 \%$ of patients, which is reflected in a median period of $12-33$ mo of progression-free survival $(4,6)$. That is, ADT prevents the progression of metastatic spread. Typically the nuclear medicine bone scan will demonstrate partial improvement or stabilization (nonprogression) of metastatic disease in response to ADT, but it is a rare occurrence for the bone scan to revert to normal (4). This observation reflects the fact that ADT rarely provides an overall cure and the likelihood of cure is inversely proportional to the extent of disease at the time of commencement of ADT. Another reason for the infrequency of a completely normal follow-up bone scan is that remodeling of bone in response to healing will mimic metastatic progression. The reversion to normal in this case is remarkable given the advanced stage of disease (Gleason score of 9) and extent of metastatic disease at the commencement of ADT.

After the progression-free survival period, there will be transformation of the disease to an androgen-independent phenotype that will not respond to ADT (6). At this point, 
alternative therapy (e.g., docetaxel) may be indicated. The mean patient survival (from commencement of ADT) is 23-37 mo (6). Androgen-independent disease is marked by disease progression despite testosterone levels below castrate levels $(<50 \mathrm{ng} / \mathrm{mL})$.

\section{CONCLUSION}

The bone scan provides a valuable tool in monitoring response to ADT because, independently of PSA levels and anatomic imaging (e.g., radiography and CT), this modality demonstrates metabolic bone changes. This case demonstrates an unusually dramatic improvement in bone scan appearance after hormone therapy in prostate cancer.

\section{DISCLOSURE}

No potential conflict of interest relevant to this article was reported.

\section{ACKNOWLEDGMENT}

We acknowledge urologist Dr. Steven Sowter for providing information to aid the formulation of the case study.

\section{REFERENCES}

1. Thorson P, Humphrey P. Minimal adenocarcinoma in prostate needle biopsy tissue. Am J Clin Pathol. 2000;114:896-909.

2. Choi S, Lee AK. Efficacy and safety of gonadotropin-releasing hormone agonists used in the treatment of prostate cancer. Drug Healthc Patient Saf. 2011;3:107119.

3. Hammerer P, Madersbacher S. Landmarks in hormonal therapy for prostate cancer. BJU Int. 2012;110:23-29.

4. Huben RP. Hormone therapy of prostatic bone metastases. Adv Exp Med Biol. 1992;324:305-316.

5. Lepor H, Shore ND. LHRH agonists for the treatment of prostate cancer: 2012. Rev Urol. 2012;14:1-12.

6. Hellerstedt BA, Pienta KJ. The current state of hormonal therapy for prostate cancer. CA Cancer J Clin. 2002;52:154-179.

7. Rossi S, ed. Australian Medicines Handbook 2012. Adelaide, Australia: Australian Medicines Handbook Pty Ltd.; 2012:591-598. 